\title{
Analysis of the Bacillus cereus SpollS antitoxin-toxin system reveals its three-component nature
}

\author{
Jana Melničáková ${ }^{\dagger}$ Zuzana Bečárová ${ }^{\dagger}$, Jana Makroczyová and Imrich Barák* \\ Institute of Molecular Biology, Slovak Academy of Sciences, Bratislava, Slovakia
}

OPEN ACCESS

Edited by:

Biswarup Mukhopadhyay,

Virginia Polytechnic Institute and State

University, USA

Reviewed by:

Xuefeng Lu,

Chinese Academy of Sciences, China

Oscar P. Kuipers,

University of Groningen, Netherlands

Marat R. Sadykov

University of Nebraska Medical

Center, USA

*Correspondence: Imrich Barák,

Institute of Molecular Biology, Slovak

Academy of Sciences, Dúbravská cesta 21, 84551 Bratislava, Slovakia

imrich.barak@savba.sk

${ }^{\dagger}$ Present Address:

Jana Melničáková,

BIOTECH s.r.o., Bratislava, Slovakia;

Zuzana Bečárová,

Institut für Molekularbiologie und

Biophysik, Zürich, Switzerland

Specialty section:

This article was submitted to Microbial Physiology and Metabolism, a section of the journal

Frontiers in Microbiology

Received: 10 May 2015

Accepted: 22 July 2015

Published: 06 August 2015

Citation:

Melničáková J, Bečárová Z, Makroczyová J and Barák I (2015) Analysis of the Bacillus cereus SpollS antitoxin-toxin system reveals its

three-component nature

Front. Microbiol. 6:808

doi: 10.3389/fmich.2015.00808
Programmed cell death in bacteria is generally associated with two-component toxin-antitoxin systems. The SpollS toxin-antitoxin system, consisting of a membrane-bound SpollSA toxin and a small, cytosolic antitoxin SpollSB, was originally identified in Bacillus subtilis. In this work we describe the Bacillus cereus SpollS system which is a three-component system, harboring an additional gene spol/SC. Its protein product serves as an antitoxin, and similarly as SpollSB, is able to bind SpollSA and abolish its toxic effect. Our results indicate that SpollSC seems to be present not only in $B$. cereus but also in other Bacilli containing a SpollS toxin-antitoxin system. In addition, we show that $B$. cereus SpollSA can form higher oligomers and we discuss the possible role of this multimerization for the protein's toxic function.

Keywords: Bacillus cereus, toxin-antitoxin system, SpollS, programmed cell death, Bacillus subtilis

\section{Introduction}

Programmed cell death (PCD) is a genetically regulated system in which a bacterial cell is able to commit suicide in response to a variety of different stresses. This response includes cell lysis or growth inhibition induced by harsh environmental conditions such as starvation or antibiotic treatment, active mother cell lysis during sporulation to release the spore, or altruistic suicide to release cell content to provide the nutrients required for the normal development of the remaining bacterial population (Engelberg-Kulka et al., 2006). PCD is usually mediated by a pair of toxin/antitoxin (TA) genes. Toxins are always highly stabile proteins. Their antidotes, the antitoxins, are usually labile proteins or small RNAs. TA systems are classified according to the nature of the antitoxin. Type I and III are small RNAs which either inhibit the synthesis of the toxin or capture it. Examples include the type I hok-sok system (Pedersen and Gerdes, 1999) and the type III ToxIN system (Fineran et al., 2009). Types II, IV, and V, on the other hand, are all proteins. They include the type II mazEF TA system (Gerdes et al., 2005), the type IV yeeU-yeeV system (Masuda et al., 2012), and the type V ghoT-ghoS system (Wang et al., 2012). These three types are distinguished based on their mode of action. The type II antitoxin is a small protein with an N-terminal DNA-binding domain and a C-terminal toxin-bonding domain, the type IV antitoxin is an antagonist of its cognate toxin and competes with it in binding to its target, and the type $\mathrm{V}$ antitoxin is an endoribonuclease that degrades the toxin-encoding mRNA (Goeders and Van Melderen, 2014).

Many bacteria harbor genes for TA systems on plasmids (Ruiz-Echevarría et al., 1995; Gerdes et al., 1997; Sayeed et al., 2000; Van Melderen, 2001; Camacho et al., 2002). These genes are part of a mechanism called post-segregational killing, which ensures that their host plasmids are retained in the daughter cells of a growing bacterial population. In this process, the stable, 
long-lived toxin kills those daughter cells which do not inherit the plasmid encoding the labile antitoxin (Gerdes et al., 1986; Lehnherr and Yarmolinsky, 1995; Hayes, 2003). Other bacterial species contain numerous toxin-antitoxin genes on their chromosome (Hayes, 2003; Tsilibaris et al., 2007; Van Melderen and Saavedra De Bast, 2009). Chromosomal TA systems may serve to prevent the spread of mobile genetic elements such as phages or plasmids; they are typically involved in the general stress response and in guarding against DNA loss (reviewed in Schuster and Bertram, 2013).

The spoIIS locus was originally identified on the Bacillus subtilis chromosome during a study of the genetic mutants that block sporulation after the formation of the polar septum (Adler et al., 2001). Formerly, the locus was thought to consist of two genes, spoIISA coding for the toxin and spoIISB for proteic antitoxin (Adler et al., 2001), thus classifying as type II TA system. A condition-dependent analysis of the transcription of all $B$. subtilis genes indicated that a third transcriptionally active region, S458, might be present in the spoIIS operon (Nicolas et al., 2012), which we name spoIISC. Inactivation of the spoIISA toxin gene has no effect on sporulation, but inactivation of the spoIISB antitoxin gene decreases sporulation efficiency by four orders of magnitude. Furthermore, disruption of spoIISA in a spoIISB null mutant restores sporulation. Thus, SpoIISB is required for sporulation only if SpoIISA is present in the cell (Adler et al., 2001). The morphological consequence of an artificially induced higher level of toxin expression is the formation of plasmolysis zones in the cytoplasmic membrane, leading to the death of the cell. The transcription of spoIISA, spoIISB, and spoIISC is upregulated during sporulation from four to up to eight hours (Nicolas et al., 2012); however, the expression of SpoIISA is independent of the crucial sporulation initiation transcription factor, Spo0A (Rešetárová et al., 2010). Production of the SpoIISA toxin is also induced during ethanol stress and nutrient deprivation. During starvation, the production of SpoIISB was detected, which suggests that SpoIISB is able to diminish the toxic effect of SpoIISA. Moreover, SpoIISB is also produced during swarming and at times of high cell density. There is presently only a little information about spoIISC, but it is known that its transcription is activated during both sporulation and biofilm formation (Nicolas et al., 2012). The SpoIISA toxin is neutralized by the formation of a tight complex with the SpoIISB antitoxin. The crystal structure of this complex revealed that SpoIISB and the cytoplasmic domain of SpoIISA form a heterotetrameric complex with C-SpoIISA 2 :SpoIISB 2 stoichiometry (Florek et al., 2011).

Homologs of SpoIISA and SpoIISB proteins have also been identified among other Bacillus species, but they display only a low level of homology. Both B. subtilis and B. cereus SpoIISA inhibit the growth of $E$. coli cells, and the SpoIISB antitoxin is able to neutralize SpoIISA toxicity in E. coli (Florek et al., 2008).

In the present study we analyze the spoIIS operon in B. cereus ATCC 14579. Even though a third trancriptionally active region in the spoIIS operon of B. subtilis was identified, it is unclear whether its product is really part of this TA system. We have found that both B. subtilis and B. cereus spoIISC encode an antitoxin that is able to diminish SpoIISA toxicity in E. coli.
As in B. subtilis, the B. cereus spoIIS operon consists of three genes: spoIISA, spoIISB, and spoIISC. Using a bacterial two hybrid system we show that $B$. cereus C-SpoIISA interacts with other C-SpoIISA molecules, as well as with SpoIISB and SpoIISC. These new positive interactions, identified in vivo, were also confirmed in vitro using a pull-down assay. In vitro analysis of the oligomeric states of $B$. cereus $C$-SpoIISA revealed that the soluble $\mathrm{C}$-SpoIISA exists in monomeric, dimeric and trimeric forms.

\section{Materials and Methods}

\section{Bacterial Strains, Growth Conditions, and Media}

The bacterial strains E. coli XL1-BLUE, DH5 $\alpha$, and MM294 were used for routine DNA manipulations. The E. coli BTH101 reporter strain was employed in the bacterial adenylate cyclasebased two-hybrid system. E. coli BL21 ( $\lambda$ DE3) cells were employed in expression of recombinant protein. E. coli cells were grown at $37^{\circ} \mathrm{C}, 28^{\circ} \mathrm{C}$ or room temperature in $\mathrm{LB}$ (Ausubel et al., 1987) or SOC medium (Hanahan, 1983) or on agar plates. When required, the medium was supplemented with appropriate antibiotics and other additives. E. coli transformation and DNA manipulations were performed using standard protocols (Sambrook et al., 1989).

\section{The Kill/Rescue Assay Cultivation}

To evaluate the effect of the expression of $B$. cereus spoIIS genes on the growth of E. coli MM294, a single colony of bacterial cells was resuspended in $100 \mu \mathrm{l}$ of LB and grown overnight on LB agar plates. The bacterial lawn was washed off with $1 \mathrm{ml} \mathrm{LB}$ and this primary culture was used to inoculate a second cell generation in LB containing $100 \mu \mathrm{g} \mathrm{ml}^{-1}$ ampicillin and $0.5 \%$ glucose $(\mathrm{w} / \mathrm{v})$. The starting optical density $\left(\mathrm{OD}_{600}\right)$ of the cell cultures was $0.05-$ 0.06 . The cells were cultivated at $37^{\circ} \mathrm{C}$ in an orbital shaker at $150 \mathrm{rpm}$ and growth was monitored by measuring the $\mathrm{OD}_{600}$ in 1 -h intervals. When the $\mathrm{OD}_{600}$ reached 0.4 , spoIIS expression was induced by the addition of 1 -arabinose to a final concentration of $0.02 \%(\mathrm{w} / \mathrm{v})$.

\section{Recombinant Plasmid Construction}

All bacterial strains and plasmids used in this study are listed in Table 1. All primers for cloning were designed for the PCR amplification of specific genes and regulatory regions and are listed in Table 2. Chromosomal DNA of B. subtilis PY79 (Youngman et al., 1984) and Bacillus cereus ATCC 14579 was used for amplification of spoIIS genes.

\section{Bacterial Two-hybrid System}

Fragments T25 and T18 from the adenylate cyclase bacterial twohybrid system (Karimova et al., 1998) were fused with the Cterminal domain of SpoIISA, full-length SpoIISB and SpoIISC, all from both $B$. cereus and B. subtilis. Chromosomal DNA from B. subtilis PY79 and B. cereus ATCC 14579 were used as PCR templates. E. coli BTH101 was used as a host for testing proteinprotein interactions. Cells were co-transformed with the relevant plasmid combinations and plated onto LB plates supplemented with $100 \mu \mathrm{g} \mathrm{ml}{ }^{-1}$ ampicillin, $30 \mu \mathrm{g} \mathrm{ml}^{-1}$ kanamycin, $40 \mu \mathrm{g}$ $\mathrm{ml}^{-1} \mathrm{X}-\mathrm{Gal}$ and $0.1 \mathrm{mM}$ IPTG and grown for $48 \mathrm{~h}$ at room temperature. 
TABLE 1 | Strains and plasmids used in this study.

\begin{tabular}{|c|c|c|}
\hline Strain & Genotype or description & Reference or origin \\
\hline \multicolumn{3}{|l|}{ E. coli } \\
\hline MM294 & $F^{-}$endA1 hsdR17 (rk- mk) supE44 thi-1 recA ${ }^{+}$ & Meselson and Yuan, 1968 \\
\hline $\mathrm{DH} 5 \alpha$ & 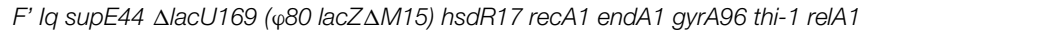 & Meselson and Yuan, 1968 \\
\hline XL1-BLUE & $\begin{array}{l}\Delta\left(\text { mcrA)183 (mcrCB-hsdSMR-mrr)173 endA1 supE44 thi-1 recA }{ }^{1} \text { gyrA96 relA lac (F' proAB lacla }\right. \\
\Delta \text { M15Tn5 kan') }\end{array}$ & Stratagene \\
\hline IB926 & pBAD24-BCIISA in MM294 & Florek et al., 2008 \\
\hline \multicolumn{3}{|c|}{ PLASMIDS USED IN KILL/RESCUE ASSAY } \\
\hline pBAD24 & Amp $^{R}$ araC; $P_{\text {BAD }}$ promoter & Guzman et al., 1995 \\
\hline pBADCIISA & $\mathrm{Amp}^{\mathrm{R}}$ araC; $\mathrm{P}_{\mathrm{BAD}}$ promoter, $B$. cereus spollSA-like gene & Florek et al., 2008 \\
\hline pBADIISAB Bc & $\mathrm{Amp}^{\mathrm{R}}$ araC; $\mathrm{P}_{\mathrm{BAD}}$ promoter, $B$. cereus spollSAB-like genes & This study \\
\hline pBADIISC Bs & $\mathrm{Amp}^{\mathrm{R}}$ araC; $\mathrm{P}_{\mathrm{BAD}}$ promoter, $B$. subtilis spollSC-like gene & This study \\
\hline pBADIISAC Bs & $\mathrm{Amp}^{R}$ araC; $\mathrm{P}_{\mathrm{BAD}}$ promoter, B. subtilis spollSAC & This study \\
\hline \multicolumn{3}{|c|}{ PLASMIDS FOR TESTING PROTEIN-PROTEIN INTERACTIONS IN VITRO } \\
\hline pET15b & Amp ${ }^{R} ;$ T7lac promoter & Novagen \\
\hline pETDuet-1 & $\mathrm{Amp}^{\mathrm{R}}$; T7lac promoter & Novagen \\
\hline pET15b-Bc-CIISA & Amp $^{R}$; T7lac promoter, B. cereus C-spollSA & Laboratory stock \\
\hline pET15b-Bc-HCIISA & Amp $^{R} ;$ T7lac promoter, His 6 tag fused with B. cereus C-spollSA & This study \\
\hline pETDuet-Bc-IISC & AmpR; T7lac promoter, B. cereus spollSC & This study \\
\hline pETDuet-Bc-HCIISAC & Amp $^{R}$; T7lac promoter, His 6 tag fused with B. cereus C-spollSA, T7lac promoter, B. cereus spollSC & This study \\
\hline pETDuetCIISA Bc & $\mathrm{Amp}^{\mathrm{R}} ;$ T7lac promoter, His 6 tag fused with B. cereus $C$-spollSA & This study \\
\hline pUT18C & Amp $^{R} ; P_{\text {lac }}$ promoter, $T 18$ & Karimova et al., 1998 \\
\hline pKT25-zip & $\mathrm{Kan}^{\mathrm{R}} ; P_{\text {lac }}$ promoter, T25 fused with zip & Karimova et al., 1998 \\
\hline pUT18C-zip & Amp ${ }^{R} ; P_{l a c}$ promoter, $T 18$ fused with zip & Karimova et al., 1998 \\
\hline pKTCIISA Bc & $\mathrm{Kan}^{\mathrm{R}} ; P_{\text {lac }}$ promoter, $T 25$ fused with $B$. cereus $C$-SpollSA & This study \\
\hline pKNTCIISA Bc & $\mathrm{Kan}^{\mathrm{R}} ; P_{\text {lac }}$ promoter, B. cereus C-spollSA fused with T25 & This study \\
\hline pUTCIISA Bc & $\mathrm{Amp}^{\mathrm{R}} ; P_{\text {lac }}$ promoter, B. cereus $C$-spol/SA fused with $T 18$ & This study \\
\hline pUTCCIISA Bc & $\mathrm{Amp}^{\mathrm{R}} ; P_{\text {lac }}$ promoter, $T 18$ fused with $B$. cereus $C$-spollSA & This study \\
\hline pUTIISB Bc & $\mathrm{Amp}^{\mathrm{R}} ; P_{\text {lac }}$ promoter, B. cereus spollSB fused with $T 18$ & This study \\
\hline pUTCIISB Bc & $\mathrm{Amp}^{\mathrm{R}} ; P_{\text {lac }}$ promoter, $T 18$ fused with $B$. cereus spollSB & This study \\
\hline pUTIISC Bc & Amp ${ }^{R} ; P_{l a c}$ promoter, B. cereus spollSC fused with $T 18$ & This study \\
\hline pUTCIISC Bc & $\mathrm{Amp}^{R} ; P_{\text {lac }}$ promoter, $T 18$ fused with $B$. cereus spollSC & This study \\
\hline pKTIISC Bc & $\mathrm{Kan}^{\mathrm{R}} ; P_{\text {lac }}$ promoter, T25 fused with $B$. cereus spollSC & This study \\
\hline pKNTIISC Bc & $\mathrm{Kan}^{\mathrm{R}} ; P_{\text {lac }}$ promoter, B. cereus spollSC fused with T25 & This study \\
\hline pKTCIISA Bs & $\mathrm{Kan}^{\mathrm{R}} ; P_{\text {lac }}$ promoter, T25 fused with $B$. subtilis $C$-spol/SA & This study \\
\hline pKNTCIISA BS & Kan $^{R} ; P_{\text {lac }}$ promoter, B. subtilis C-spol/SA fused with T25 & This study \\
\hline pUTCIISA Bs & Amp ${ }^{R} ; P_{\text {lac }}$ promoter, B. subtilis C-spollSA fused with $T 18$ & This study \\
\hline pUTCCIISA Bs & $\mathrm{Amp}^{\mathrm{R}} ; P_{\text {lac }}$ promoter, $T 18$ fused with $B$. subtilis $C$-spol/SA & This study \\
\hline pUTIISB Bs & Amp $^{R} ; P_{l a c}$ promoter, B. subtilis spol/SB fused with $T 18$ & This study \\
\hline
\end{tabular}


TABLE 1 | Continued

\begin{tabular}{lll}
\hline Strain & Genotype or description & Reference or origin \\
\hline pUTCIISB Bs & $A m p^{R} ; P_{l a c}$ promoter, T18 fused with B. subtilis spollSB & This study \\
pUTIISC Bs & $A m p^{R} ; P_{l a c}$ promoter, B. subtilis spollSC fused with T18 & This study \\
pUTCIISC Bs & $A m p^{R} ; P_{l a c}$ promoter, T18 fused with B. subtilis spollSC & This study \\
pKTIIISC Bs & $\mathrm{Kan}^{\mathrm{R}} ; P_{l a c}$ promoter, T25 fused with B. subtilis spollSC & This study \\
pKNTIISC Bs & $\mathrm{Kan}^{\mathrm{R}} ; P_{l a c}$ promoter, B. subtilis spollSC fused with T25 & This study \\
\hline
\end{tabular}

TABLE 2 | Primers used in this study.

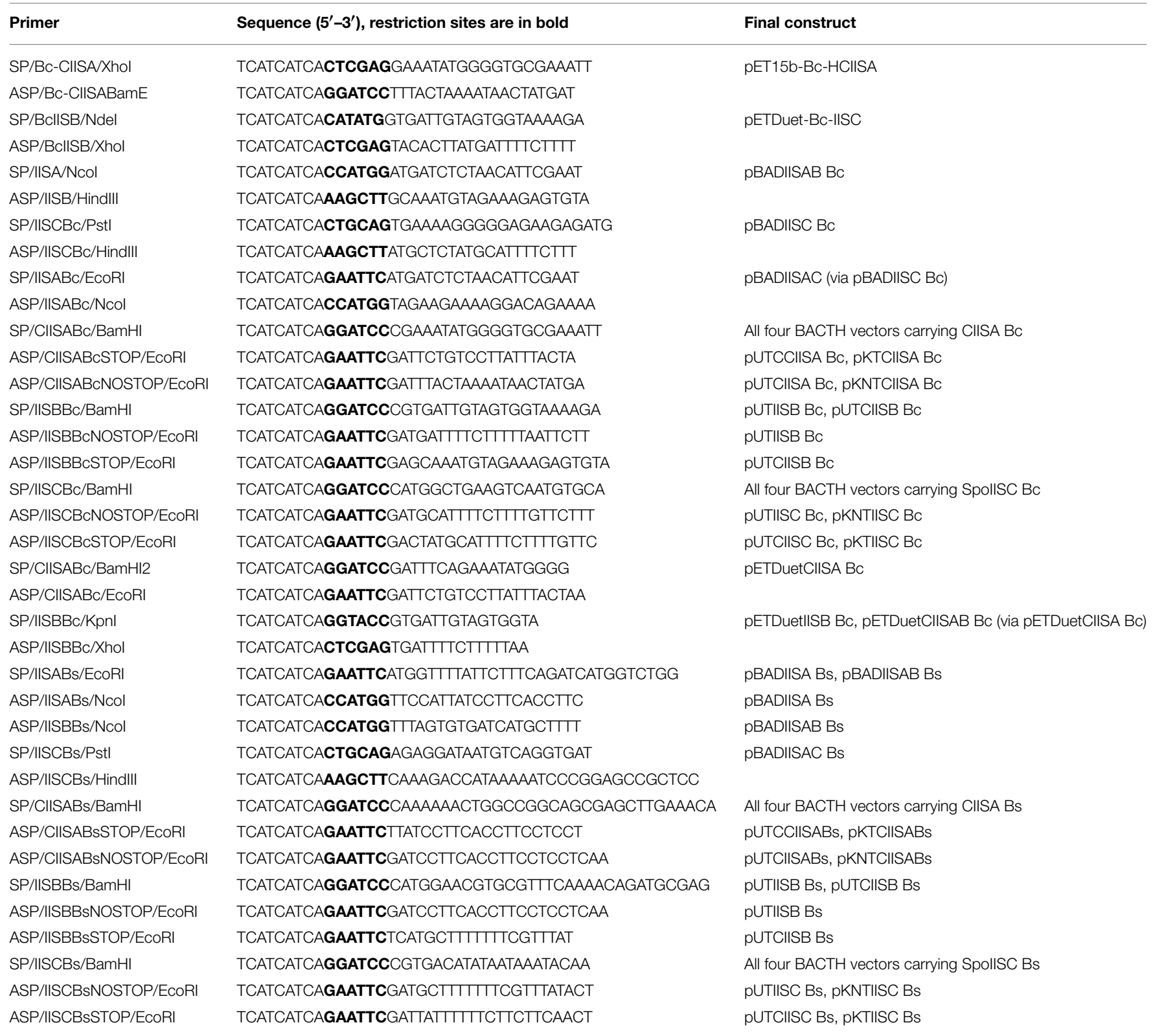

\section{SDS-PAGE Analysis}

One dimensional SDS-PAGE was performed according to Laemmli (1970). Samples of whole cell lysates of recombinantprotein expressing E. coli BL21 ( $\lambda \mathrm{DE} 3)$ cells, protein complexes, or purified protein samples were resuspended in sample buffer [4\% SDS (w/v); $10 \% \beta$-mercaptoethanol (v/v); $20 \%$ glycerol (v/v); $0.25 \mathrm{M}$ Tris-Cl, $\mathrm{pH} 8$ ] and boiled for $10 \mathrm{~min}$. Denatured proteins were separated in $12 \%$ polyacrylamide gels. Due to the low 
molecular weight of B. cereus SpoIISC $(6.6 \mathrm{kDa})$, this protein was analyzed using $16.5 \%$ Tricine-SDS-PAGE (Schägger and von Jagow, 1987), which better resolves such small proteins. As for the SDS-PAGE, samples of whole cell lysates of $E$. coli BL21 ( $\lambda$ DE3) cells expressing recombinant SpoIISC and purified protein samples were resuspended in Novex sample buffer (Invitrogen, USA), then heated for $5 \mathrm{~min}$ in a boiling water bath and briefly spun down. The gels were run at $25 \mathrm{~mA}$ and stained with Coomassie brilliant blue R-250.

\section{Pull-down Assay}

Pull-down assays were used to confirm in vitro the interactions between B. cereus C-SpoIISA and SpoIISB, SpoIISC and B. subtilis C-SpoIISA. In order to investigate the interaction of His $_{6}$-tagged B. cereus C-SpoIISA with S-tagged SpoIISB, the following proteins were isolated: $\mathrm{His}_{6}$-tagged C-SpoIISA, Stagged SpoIISB and $\mathrm{His}_{6}$-tagged C-SpoIISA expressed together with S-tagged SpoIISB. E. coli BL21 ( $\lambda \mathrm{DE} 3$ ) competent cells were transformed with the pETDuetCIISA Bc and pETDuetIISB $\mathrm{Bc}$ plasmids (Table 1) for the overexpression of $\mathrm{His}_{6}$-tagged C-SpoIISA and S-tagged SpoIISB, respectively. Transformation

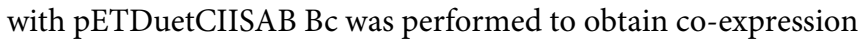
of His $_{6}$-tagged C-SpoIISA with S-tagged SpoIISB. The resulting cell cultures were grown at $28^{\circ} \mathrm{C}$ in $\mathrm{LB}$ medium supplemented with $100 \mu \mathrm{g} \mathrm{ml}^{-1}$ ampicillin and $0.5 \%$ glucose. Recombinant protein expression was induced by the addition of IPTG to a final concentration of $0.5 \mathrm{mM}$, when the culture reached an $\mathrm{OD}_{600}$ of $\sim 0.6$. Cells were harvested $5 \mathrm{~h}$ after induction, centrifuged, and resuspended in solubilization buffer $[20 \mathrm{mM}$ Tris- $\mathrm{Cl}, \mathrm{pH} 8$; $150 \mathrm{mM} \mathrm{NaCl} ; 10 \%$ glycerol (v/v); $10 \mathrm{mM} \mathrm{MgCl} 2 ; 1 \mathrm{mM}$ AEBSF]. Proteins were solubilized by overnight incubation at $14^{\circ} \mathrm{C}$ in the presence of $10 \mathrm{mM}$ CHAPS (Sigma Aldrich). Samples were centrifuged for $30 \mathrm{~min}$ at $60000 \times \mathrm{g}$ and $4^{\circ} \mathrm{C}$. Soluble fractions were loaded onto a Ni Sepharose HP column (Amersham Bioscience) and washed; bound proteins were eluted with an imidazole step gradient from $0.2 \mathrm{M}$, to $0.4 \mathrm{M}, 0.6 \mathrm{M}$ and $1 \mathrm{M}$. The most concentrated fraction of the $\mathrm{His}_{6}$-tagged C-SpoIISA, that with $1 \mathrm{M}$ imidazole, was used in further experiments. The S-tagged B. cereus SpoIISB $0.2 \mathrm{M}$ imidazole fraction was used as a control for non-specific binding to the Ni column. Finally, the $0.4 \mathrm{M}$ imidazole fraction of SpoIISB was used in the assay to confirm that $\mathrm{His}_{6}$-tagged C-SpoIISA interacts with S-tagged SpoIISB. These proteins and the C-SpoIISA-SpoIISB protein complex were fractionated by $16.5 \%$ Tricine-SDS-PAGE. The fractioned proteins were transferred onto a nitrocellulose membrane and subsequently Western blotted.

The pull-down assay of $\mathrm{His}_{6}$-tagged B. cereus $\mathrm{C}$-SpoIISA with untagged SpoIISC was performed similarly as described above. In this case, $E$. coli BL21 ( $\lambda \mathrm{DE} 3$ ) cells were transformed with pETDuet-Bc-HCIISAC for the interaction study and pETDuet$\mathrm{Bc}$-IISC (Table 1) to control for the non-specific binding of B. cereus SpoIISC to the Ni column.

\section{Glutaraldehyde Crosslinking}

The oligomeric state of recombinant B. cereus $\mathrm{His}_{6}$-C-SpoIISA was assessed by glutaraldehyde crosslinking. E. coli BL21 ( $\lambda$ DE3) competent cells were transformed with pETDuetCIISA Bc, and protein expression was induced with $0.5 \mathrm{mM}$ IPTG for $5 \mathrm{~h}$ at $28^{\circ} \mathrm{C}$. Cells were then harvested and resuspended in a buffer containing $20 \mathrm{mM}$ HEPES $\mathrm{pH} 7.5$ and $150 \mathrm{mM} \mathrm{NaCl}$ and sonicated. The soluble fractions were centrifuged for $30 \mathrm{~min}$ at $60000 \times \mathrm{g}$ and $4^{\circ} \mathrm{C}$ and then loaded onto a Ni Sepharose HP column (Amersham Bioscience). Proteins were eluted with an imidizole step gradient from $0.1 \mathrm{M}$ to $0.2 \mathrm{M}, 0.3 \mathrm{M}$ and $1 \mathrm{M}$. For the crosslinking, $80 \mu \mathrm{g}$ of protein was mixed with $5 \mu \mathrm{l}$ of a freshly prepared solution of $2.3 \%$ glutaraldehyde to make a total volume of $100 \mu$ l. This reaction mixture was incubated for $30 \mathrm{~min}$ at $37^{\circ} \mathrm{C}$ and the reaction was then stopped by the addition of $10 \mu \mathrm{l}$ of $1 \mathrm{M}$ Tris- $\mathrm{HCl}, \mathrm{pH}$ 8.0. The crosslinked molecules of B. cereus CSpoIISA were loaded onto a $12 \%$ SDS-PAGE gel and detected by Western blotting.

\section{Western Blotting}

To visualize the interaction of $B$. cereus C-SpoIISA with the heterologous B. subtilis C-SpoIISA as well as the interaction of B. cereus C-SpoIISA with SpoIISB, we performed Western blotting using the general protocol of Ausubel et al. (1987). Briefly, proteins were fractionated by either $12 \%$ SDS-PAGE or 16.5\% Tricine-SDS-PAGE and transferred onto a nitrocellulose membrane (Hybond ECL; Amersham Bioscience). To prevent non-specific binding, the membrane was treated using $5 \%$ nonfat milk in Tris-buffered saline with $0.05 \%$ Tween $20(\mathrm{v} / \mathrm{v})$. $\mathrm{His}_{6}$-tagged B. cereus C-SpoIISA was probed with an anti His $_{6}$-tag monoclonal antibody (Novagen; catalog no. 70796-3) while S-tagged B. subtilis C-SpoIISA and S-tagged B. cereus SpoIISB were probed with an anti S-tag monoclonal antibody (Novagen; catalog no. 71549-3). Protein interactions were detected using antimouse horseradish peroxidase-conjugated secondary antibodies (Promega; catalog no. W402B).

\section{Gel Filtration}

To analyze the oligomerization of $B$. cereus C-SpoIISA using gel filtration, we developed a procedure for purifying untagged $B$. cereus C-SpoIISA. First, E. coli BL21 ( $\lambda \mathrm{DE} 3$ ) cells were transformed with the plasmid pET15b-Bc-CIISA. Next, the cell culture was grown at $28^{\circ} \mathrm{C}$ in $\mathrm{LB}$ medium supplemented with $100 \mu \mathrm{g} \mathrm{ml}^{-1}$ ampicillin. When the culture reached an $\mathrm{OD}_{600}$ of 0.6 , the expression of untagged C-SpoIISA was induced with $0.5 \mathrm{mM}$ IPTG. The cells were harvested $5 \mathrm{~h}$ after induction, centrifuged and resuspended in a resuspension buffer containing $50 \mathrm{mM}$ glycine, $\mathrm{pH} 10 ; 50 \mathrm{mM} \mathrm{NaCl} ; 10 \mathrm{mM} \mathrm{MgCl}_{2} ; 10 \%$ glycerol $(\mathrm{v} / \mathrm{v})$; and $1 \mathrm{mM}$ AEBSF. The protein was solubilized by incubating at $14^{\circ} \mathrm{C}$ overnight in the presence of $10 \mathrm{mM}$ CHAPS (Sigma Aldrich). The soluble fractions were cleared by centrifugation for $30 \mathrm{~min}$ at $60000 \times \mathrm{g}$ and $4^{\circ} \mathrm{C}$ and loaded onto a HiPrep DEAE Sepharose FF 16/10 column (GE Healthcare Life Sciences), which had previously been equilibrated with a resuspension buffer containing $10 \mathrm{mM}$ CHAPS. The protein eluted in the flow-through fraction and was loaded onto a HiPrep Q Sepharose HP 16/10 column (GE Healthcare Life Sciences), previously equilibrated with the same solution. The protein was eluted from this column with a continuous salt gradient ranging from 0.2 to $1 \mathrm{M} \mathrm{NaCl}$. The purified protein was applied to a Superose 6 10/300 GL column (GE Healthcare Life Sciences) 
connected to an FPLC (GE Healthcare Life Sciences) instrument controlled by UNICORN 5.11 software, at a flow rate of $0.4 \mathrm{ml}$ $\mathrm{min}^{-1}$. The elution was followed using UV absorbance at $280 \mathrm{~nm}$.

\section{Dynamic Light Scattering Measurements}

DLS experiments were performed at $20^{\circ} \mathrm{C}$ on a Zetasizer Nano ZS instrument (Malvern Instrument) controlled by DTS software (version 5.1, Malvern Instruments Ltd). The instrument has a $90^{\circ}$ scattering angle. The purified protein, at a concentration of $100 \mu \mathrm{M}$ in a resuspension buffer at $\mathrm{pH} 8$ containing $10 \mathrm{mM}$ CHAPS, was filtered through $20 \mathrm{~nm}$ filters into a $40 \mu \mathrm{l}$ cuvette. A single measurement consisted of 20 runs of $12 \mathrm{~s}$ each. All measurements were done in triplicate. The samples gave a clear signal (the $y$-intercept was 0.95 ) and required only moderate attenuation (set at 7).

\section{Bioinformatics Analysis}

Promoter analysis was performed using BPROM (Solovyev and Salamov, 2011). Identification of Rho-independent bacterial terminators was done using was done using ARNold web tool (Naville et al., 2011; http://rna.igmors.u-psud.fr/toolbox/ arnold/). B. cereus SpoIISA membrane topology prediction was done using the MEMSAT3 and MEMSAT-SVM algorithms (http://bioinf.cs.ucl.ac.uk/psipred/; Nugent and Jones, 2009).

\section{Results and Discussion}

\section{The SpollSABC Toxin-antitoxin System}

The SpoIIS toxin-antitoxin system in Bacillus subtilis consists of a SpoIISA toxin that is neutralized by a SpoIISB antitoxin (Adler et al., 2001; Florek et al., 2008). However, profiling of the condition-dependent transcription of $B$. subtilis revealed the presence of a third transcriptionally active region, denoted as S458 (Nicolas et al., 2012), located 55 bp downstream of spoIISB in the spoIIS operon, which we named spoIISC. Adler et al. (2001) identified two promoters in the B. subtilis spoIIS operon. The first promoter $\left(\mathrm{P}_{\mathrm{A}}\right)$ is located upstream of spoIISA and is important for regulating the expression of both spoIISA and spoIISB. The second promoter $\left(\mathrm{P}_{\mathrm{B}}\right)$ is located within the spoIISA gene and serves to regulate the expression of spoIISB. A promoter search using BPROM (see Materials and Methods) revealed a possible additional promoter $\left(\mathrm{P}_{C}\right)$ downstream of spoIISB which could be used to regulate the expression of spoIISC. Its - 35 sequence is $5^{\prime}$-TTCCTT- $3^{\prime}$ and its -10 sequence is $5^{\prime}$-ACATATAAT- $3^{\prime}$. In addition, a search for Rho-independent bacterial terminators using the ARNold tool identified the terminator (5' - GAAAAA ATAAATCCCGGAGCGGCTCCGGGATTTTTATGGTCT - $3^{\prime}$; letters in bold indicates bases contributing to the loop structure, underlined letters are bases forming the stem of terminator hairpin) immediately after the spoIISC STOP codon.

We previously found that a two-component SpoIIS system also exists in B. cereus (Florek et al., 2008). The position of its locus on the chromosome is completely different from that of the spoIIS operon in B. subtilis. While the B. subtilis spoIIS operon is $115^{\circ}$ away from the origin of replication, the $B$. cereus spoIIS locus is $158^{\circ}$ away. The B. cereus spoIIS operon consists of spoIISA (BC_2436), which encodes a 245-residue SpoIISA-like protein, and BC_2437, which encodes a hypothetical protein with 58 residues. As shown in Florek et al. (2008), BC_2437 is found 316 bp downstream of the spoIISA-like gene and was named spoIISB since its SpoIISB-like product was able to neutralize the toxicity of the SpoIISA-like protein in E. coli. Prompted by the identification of a putative third transcript in the B. subtilis spoIIS operon (Nicolas et al., 2012), we revisited the bioinformatics analysis of the B. cereus spoIIS operon and found that the B. cereus spoIIS operon also likely contains three genes: the BC_2436 ORF encoding a 245-residue SpoIISA-like protein; a 138-bp ORF 103 bp downstream of this gene, which encodes a 45-residue, putative SpoIISB; and a further 72 bp downstream of that, the BC_2437 ORF, which encodes the 58-residue protein we had previously called SpoIISB, but which we now call SpoIISC (Figure 1; Florek et al., 2008). As in the B. subtilis analysis, BPROM identified putative promoters in this operon. B. cereus spoIISA appears to be driven by the putative promoter $\mathrm{P}_{A}$, the putative $\mathrm{P}_{B}$ promoter for controlling spoIISB gene expression is found within the spoIISA gene, and the putative $\mathrm{P}_{\mathrm{C}}$ promoter that likely regulates the expression of spoIISC is located downstream of the spoIISB gene. ARNold tool predicts that a Rho-independent bacterial transcription terminator, with the sequence 5/-AAAGAACA AAAGAAAATGCATAGAGCATTTTCTTTTGTTTTTTTA-3' (letters in bold indicates bases contributing to the loop structure, underlined letters are bases forming the stem of terminator hairpin). This sequence overlaps with the end of $B$. cereus spoIISC gene (Figure 1A).

The presence of three promoters in the spoIIS locus may be due to the different conditions under which the expression of individual genes is induced. The transcription of all three $B$. subtilis spoIIS genes is clearly induced during sporulation, but during nutrient deprivation only the spoIISA and spoIISB genes are transcribed (Nicolas et al., 2012). Moreover, there are conditions which induce transcription of only one of these genes: spoIISA is transcribed during ethanol stress, spoIISB during swarming and at high cell density, and spoIISC during biofilm formation (Nicolas et al., 2012).

Both $B$. subtilis and B. cereus SpoIISA-SpoIISB systems are clear examples of type II TA systems (Adler et al., 2001; Florek et al., 2011). The spoIIS operon has been identified only in Bacilli, and only a low level of homology can be detected between the SpoIIS proteins of B. subtilis and B. cereus (Florek et al., 2008). SpoIISA proteins display $17.3 \%$ identity and $30.2 \%$ similarity, while the SpoIISB proteins have only $12.5 \%$ identity and $17.9 \%$ similarity. The SpoIISC proteins have the lowest homology, with only $8.6 \%$ identity and $15.5 \%$ similarity. On the other hand, the SpoIISB and SpoIISC proteins from one of these organisms exhibit a higher level of homology with each other than with their counterparts in the other organism. Thus the B. subtilis SpoIISB and SpoIISC proteins show $37.5 \%$ homology and $12.5 \%$ identity while $B$. cereus SpoIISB and SpoIISC have $35.6 \%$ similarity and $27.1 \%$ identity (Figure 1B).

Bacterial type II TA systems are normally organized so that the first gene in the operon codes for the antitoxin and the toxin is positioned farther downstream; both genes are usually preceded by their own promoters. This arrangement ensures an abundance of antitoxin is produced to prevent toxin activity when it is 


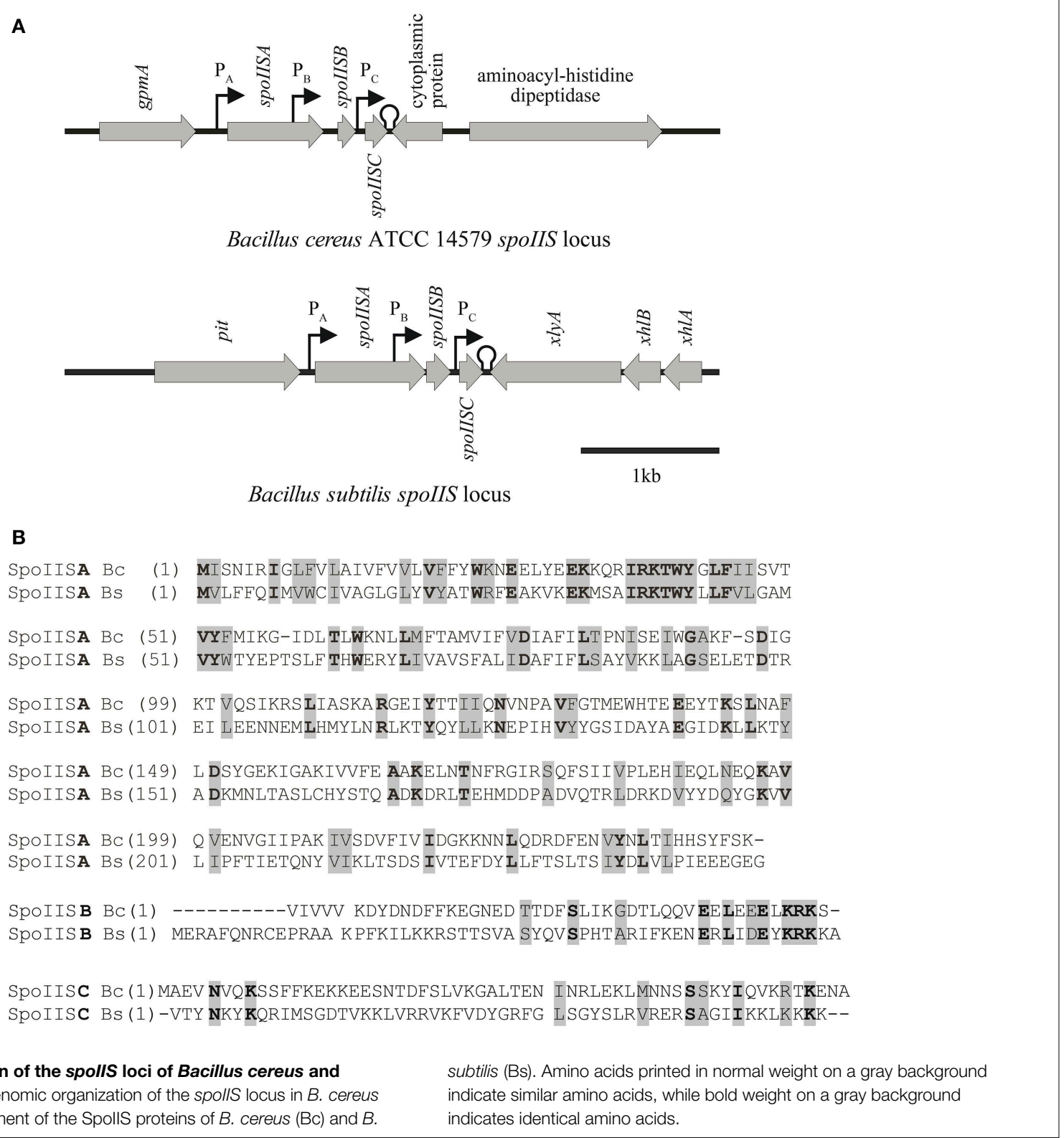

FIGURE 1 | Comparison of the spollS loci of Bacillus cereus and Bacillus subtilis. (A) Genomic organization of the spollS locus in $B$. cereus and $B$. subtilis. (B) Alignment of the SpollS proteins of $B$. cereus $(\mathrm{Bc})$ and $B$. indicate similar amino acids, while bold weight on a gray background indicates identical amino acids. undesirable. One exception to this arrangement is the higBA TA module in pathogenic Proteus species (Hurley and Woychik, 2009). As noted above, the spoIIS system is another, with the toxin preceding its two putative antitoxin genes. The SpoIIS TA system is unusual in another way as well. The typical type II TA system is a two-component system, but the SpoIIS TA system consists of three components: the SpoIISA toxin, the SpoIISB antitoxin and the third component SpoIISC (a likely antitoxin). Other threecomponent type II TA systems have previously been reported, including the $\omega-\varepsilon-\zeta$ TA module encoded by the Streptococcus pyogenes plasmid pSM19035, the paaR-paaA-parE TA module encoded by E. coli O157:H7, and the pasA/pasB/pasC module of the Thiobacillus ferrooxidans plasmid pTF-FC2 (reviewed in Unterholzner et al., 2013). In all of these systems, at least one of the three components is involved in autoregulating the operon.
There is presently no information about whether the expression of the spoIIS operon in Bacilli is autoregulated.

\section{The spolISB and spolISC Genes Encode Antitoxins in Bacillus Cereus}

B. subtilis transcription analysis by Nicolas et al. (2012) and in this study have revealed that the spoIIS operon is formed by the spoIISA, spoIISB, and spoIISC genes. In E. coli, B. subtilis SpoIISA inhibited bacterial growth and SpoIISB was able to neutralize SpoIISA toxicity (Florek et al., 2008). Previously, we observed that B. cereus SpoIISA, like B. subtilis SpoIISA, has a toxic effect on $E$. coli growth (Florek et al., 2008), but at that time, we had incorrectly designated ORF BC_2437 as spoIISB. A new bioinformatics analysis, prompted by the likely existence of a third gene in the B. subtilis spoIIS operon by Nicolas et al. 
(2012), shows that BC_2437 indeed contains spoIISC and that SpoIISB is a 45 -residue protein of unknown function encoded by a small ORF (only $138 \mathrm{bp}$ ) located between the spoIISA and spoIISC genes.

To determine if $B$. cereus SpoIISB and SpoIISC are both able to neutralize the toxicity of $B$. cereus SpoIISA in E. coli, the corresponding genes spoIISAB and spoIISAC were cloned into pBAD24 vectors under the control of arabinose-inducible $\mathrm{P}_{\mathrm{BAD}}$ promoters to generate $\mathrm{pBADIISAB} \mathrm{Bc}$ and $\mathrm{pBADIISAC} \mathrm{Bc}$. These plasmids were subsequently introduced into E. coli MM294 cells. The growth of these transformed cells, together with the control strains IB890 (E. coli MM294 / pBAD24) and IB926 (E. coli MM294/pBAD-BCIISA) (Florek et al., 2008), was monitored after the induction of protein expression. As found previously (Florek et al., 2008), the growth of E. coli cells expressing only B. cereus SpoIISA was inhibited. On the other hand, both SpoIISB and SpoIISC were able to neutralize the toxicity of SpoIISA: the growth curves of those strains which expressed both SpoIISA and either the SpoIISB antitoxin or SpoIISC were similar to that of the wild-type IB890 E. coli cells (Figure 2A). Because B. cereus SpoIISB and SpoIISC disturb SpoIISA toxicity when expressed in $E$. coli cells, it can be concluded that both spoIISB and spoIISC encode antitoxins and that they are likely to have similar functions as the antitoxins in $B$. subtilis. Indeed, an identical set of experiments using the B. subtilis genes rather than the $B$. cereus ones gives very similar results (Figure 2B).

\section{The Interactions of SpollS Proteins in a Bacterial Two Hybrid System}

The clearest evidence that B. subtilis SpoIISA and SpoIISB directly interact can be found in the crystal structure of the Cterminal domain of SpoIISA in complex with SpoIISB (Florek et al., 2011). To analyze the protein-protein interactions of the $B$. cereus SpoIIS proteins in vivo, we made use of the bacterial adenylate cyclase two hybrid system (Karimova et al., 1998). Like B. subtilis SpoIISA, B. cereus SpoIISA is predicted to be a membrane protein with three membrane-spanning segments. However, we decided to use only the cytoplasmic domains in this protein-protein interaction study, since the whole SpoIISA protein is toxic for $E$. coli as we have shown previously. We prepared fusions of the C-terminal domain of B. cereus SpoIISA, SpoIISB, and SpoIISC with the adenylate cyclase fragments T25 and T18. All possible interactions were tested and compared with those of similar SpoIIS fusion proteins from B. subtilis (Figure 3).

Our results confirmed the dimerization of $B$. subtilis CSpoIISA as well as the interaction of B. subtilis C-SpoIISA with SpoIISB described in Florek et al. (2011). A positive interaction was also observed for B. subtilis C-SpoIISA with SpoIISC (Figure 3). Finally, we found that the B. cereus Cterminal domain of SpoIISA can interact with another C-SpoIISA protomer, with SpoIISB and with SpoIISC (Figure 3).

\section{B. cereus SpolISB and SpollSC are Able to Bind the C-terminal Domain of SpollSA in vitro}

To analyze these protein-protein interactions in vitro, we prepared three derivatives of the pETDuet recombinant expression plasmid, each containing one of the following genes, all under the control of an IPTG-inducible T7 promoter: a gene coding for a His $_{6}$-tagged B. cereus $\mathrm{C}$-SpoIISA, an S-tagged SpoIISB and an untagged SpoIISC (Table 1). We found that $\mathrm{His}_{6}$-tagged C-SpoIISA binds the Ni column and that S-tagged SpoIISB and untagged SpoIISC creates a tight complex with C-SpoIISA which can be eluted by a solubilization buffer step gradient containing 0.1-1 mM imidazole (Figure 4).

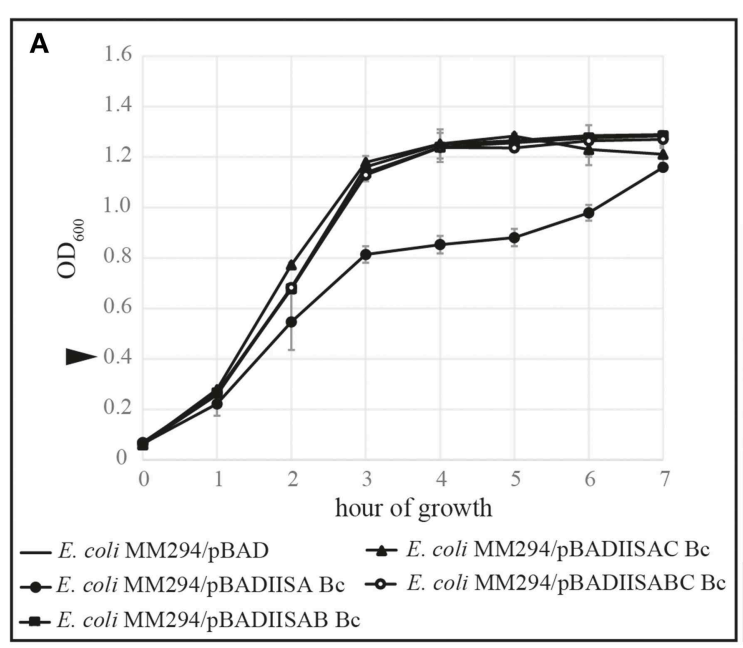

FIGURE 2 | Kill/rescue assay in E. coli MM294. In order to test the ability of SpollSC to act as an antitoxin for SpollSA, SpollS proteins were expressed alone or in combination in E. coli cells. All results are mean values of three independent replicates and the bars represent 1 SD. The growth of E. coli cells expressing the SpollSA toxin (circle) was inhibited while those

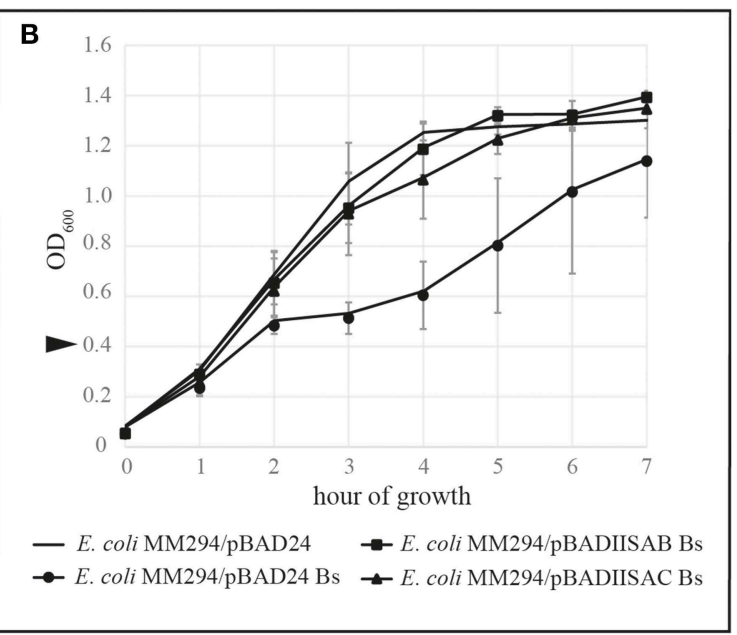

cells expressing either the SpollSAB complex (square) or the SpollSAC complex (triangle) had wild-type growth (no marker). Arrows indicate the addition of $0.02 \%$ arabinose to induce expression. (A) The effect of the $B$. cereus SpollS proteins on the growth of $E$. coli MM294 cells. (B) The effect of the B. subtilis SpollS proteins on the growth of $E$. coli MM294 cells. 


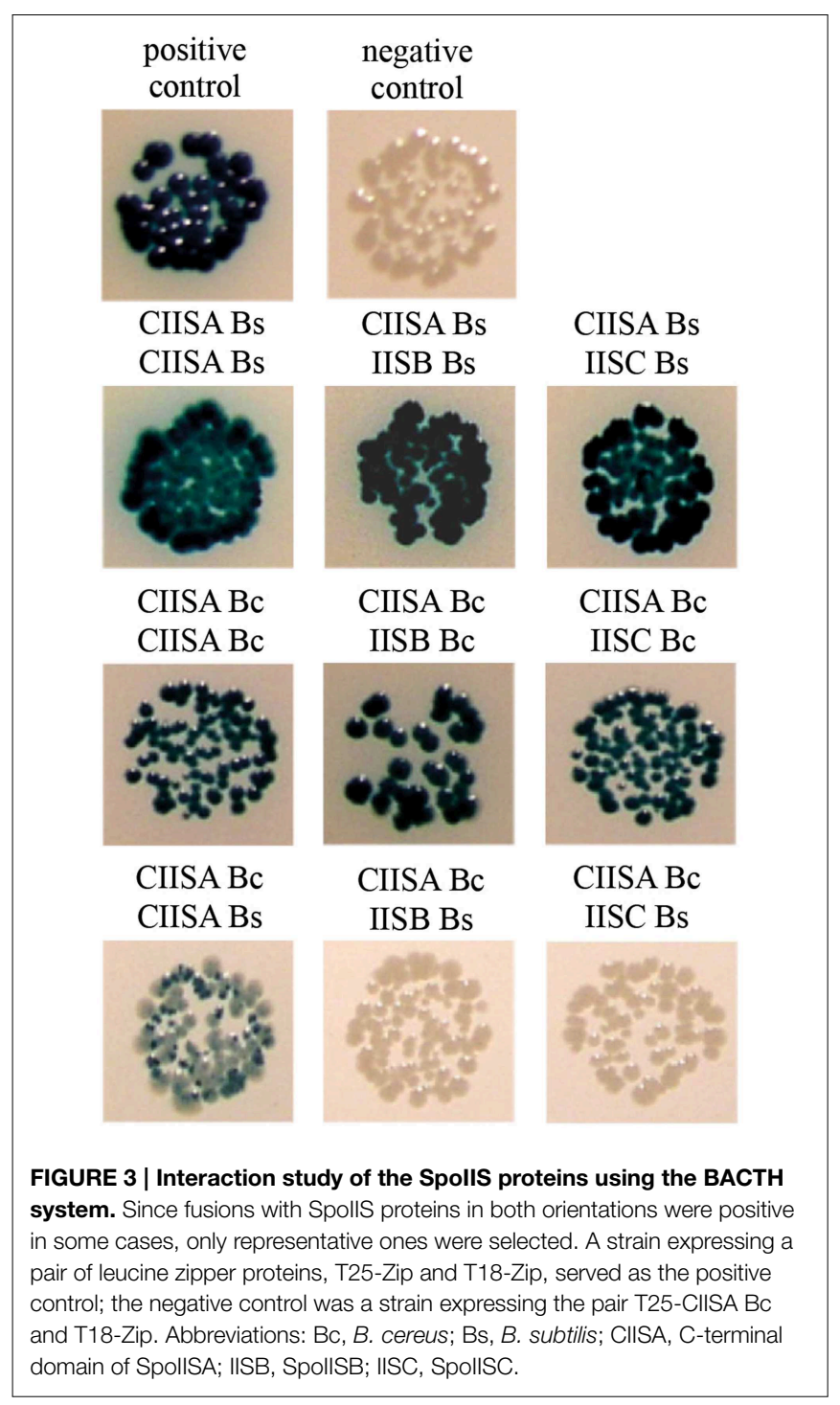

The interaction of B. cereus His $_{6}$-tagged C-SpoIISA with Stagged SpoIISB was confirmed in a pull-down assay by the coelution of both proteins from a Ni column. When SpoIISB is co-expressed in E. coli together with $\mathrm{His}_{6}$-tagged C-SpoIISA, the $\mathrm{His}_{6}$-tagged C-SpoIISA binds the Ni column, and since S-tagged SpoIISB binds C-SpoIISA, the two are pulled down together as a complex during elution with $0.4 \mathrm{M}$ imidazole (Figure 4A). This complex could be detected by Western blotting using an anti$\mathrm{His}_{6}$-tag monoclonal antibody to identify His ${ }_{6}$-tagged C-SpoIISA (Figure 4A, lane 3) and an anti-S-tag monoclonal antibody to identify the S-tagged SpoIISB (Figure 4A, lane 6).

A similar approach was used to test the interaction of untagged B. cereus SpoIISC with $\mathrm{His}_{6}$-tagged C-SpoIISA in vitro (Figure 4B). B. cereus SpoIISC expressed in E. coli BL21 (DE3) appears in the insoluble fraction of the cell lysate according to $16.5 \%$ Tricine/SDS-PAGE (data not shown). However, when coexpressed with $B$. cereus $\mathrm{His}_{6}$-tagged C-SpoIISA in the same cells, they form a complex which is able to pull SpoIISC out of the insoluble fraction. The whole complex can then be solubilized and purified from the soluble fraction by affinity chromatography.

\section{B. cereus C-terminal Domain of SpolISA forms an Oligomer}

The crystal structure of the B. subtilis SpoIISA C-terminal domain shows that the protein dimerizes by forming a four-helix bundle using the first and last $\alpha$-helices of each molecule (Florek et al., 2011). Our bacterial two-hybrid experiments showed that $B$. cereus C-SpoIISA interacts with other B. cereus C-SpoIISA molecules (Figure 3), suggesting that this molecule also forms oligomers. The oligomeric form of C-SpoIISA was examined by measuring the hydrodynamic radius of dissolved particles using dynamic light scattering. A cumulant analysis showed that the sample was monomodal (i.e., had only one peak, Figure 5A), and was polydisperse, with a polydispersity index of 0.255 and an overall polydispersity of $50.32 \%$. The polydispersity indicates broader particle size distribution, and thus the hydrodynamic radius and corresponding molecular mass cannot be reliably calculated.

The SpoIISA oligomerisation was examined further by sizeexclusion chromatography of C-SpoIISA using a Superose 6 $10 / 300 \mathrm{GL}$ column. In this analysis, most of the protein appeared in the void volume fraction of the column, which was determined from the elution of Blue dextran 2000 ( 2000 kDa, Pharmacia) (Figure 5B). Three small peaks were detected, however, and likely correspond to the $59.1 \mathrm{kDa}$ trimer, the $39.4 \mathrm{kDa}$ dimer and the 19.7 kDa monomer of C-SpoIISA. The existence of monomeric, dimeric and trimeric states was confirmed by glutaraldehyde crosslinking (Figure 5C), but the existence of higher oligomeric forms could not be confirmed because such large species would not have been able to enter the crosslinking gel.

Taken together, the above results indicate that B. cereus $\mathrm{C}$ SpoIISA is able to form higher multimers, even if their nature is unclear. In this respect, its behavior differs from that of B. subtilis C-SpoIISA, which formed only dimers (Florek et al., 2011). Whole B. subtilis SpoIISA does seem to form higher oligomers, but this seems to require its $\mathrm{N}$-terminal transmembrane domain rather than just its C-terminal cytosolic domain (Makroczyová et al., 2014). Earlier studies also suggested that whole SpoIISA oligomerizes, and moreover suggested that it forms holin-like pores (Adler et al., 2001). Whether either the B. subtilis or B. cereus proteins actually do form such pores remains unknown, however.

Finally, this study describes the SpoIISC protein, a third component of the spoIIS locus. This protein serves as an antitoxin and shows similarity to SpoIISB. The presence of two antitoxin genes in the spoIIS locus of both B. subtilis and B. cereus naturally poses the question of the role of such duplication. One possibility is that the different proteins are linked to different conditions under which they might be expressed, as was shown for $B$. subtilis SpoIIS system (Nicolas et al., 2012). They may also act as transcription regulators, as some other antitoxins are known to. It is also possible that their different amino-acid compositions could affect their affinity for SpoIISA, leading to different degrees of inhibition. In any case, our results show that the SpoIIS TA system is much more complex than had previously been thought. 


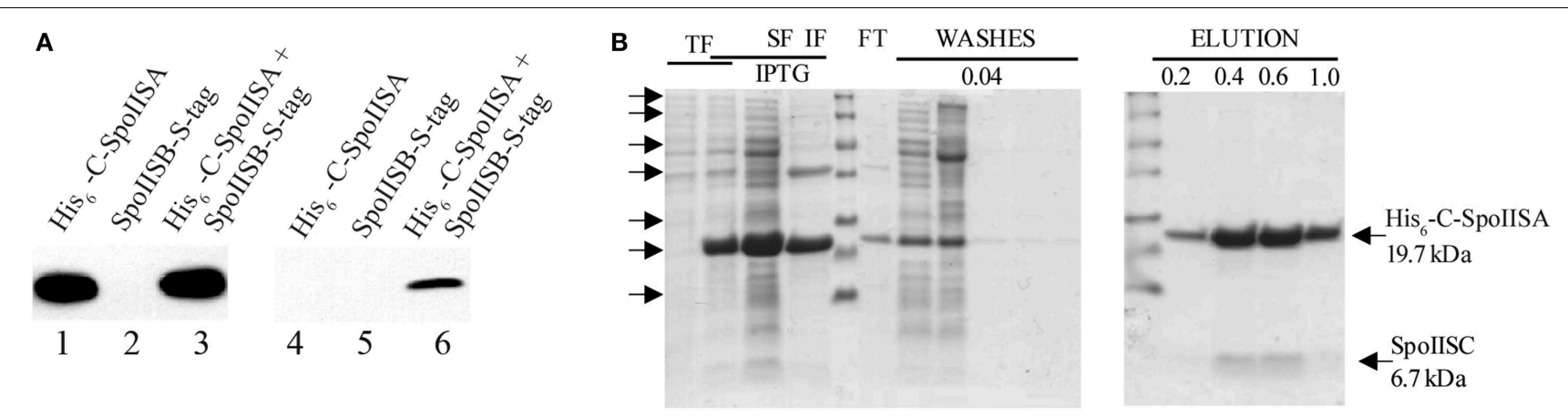

FIGURE 4 | Pull-down assays of B. cereus SpollSB and SpolISC with C-SpolISA. The soluble fractions of lysed bacterial cells were applied to a $\mathrm{Ni}$ Sepharose HP column. The eluted proteins were identified by Western blotting (A) and Coomassie brilliant blue R-250 staining (B). (A) In the Western blot, the eluted proteins were probed with an anti-His 6 monoclonal antibody (lanes 1-3) or with an anti-S monoclonal antibody (lanes 4-6). Lanes 1 and 4 contain purified His $_{6}$-tagged C-SpollSA, lanes 2 and 5, purified S-tagged SpollSB. S-tagged SpollSB does not bind a Ni Sepharose
HP column. Lanes 3 and 6 show that His ${ }_{6}$-tagged C-SpollSA can pull down S-tagged SpollSB and therefore that there is an interaction between them. (B) A pull-down assay showing an interaction between $\mathrm{His}_{6}$-tagged C-SpollSA and SpolISC when both proteins are co-expressed. TF, total fraction; SF, soluble fraction; IF, insoluble fraction; FT, flow-through fraction, $0.04 ; 0.2 ; 0.4,0.6$, and 1.0-molarity of imidazole used in washing and elution. The arrows mark the following positions on the protein ladder from top to bottom: $116,66.2,45,35,25,18.4$, and $14.4 \mathrm{kDa}$.
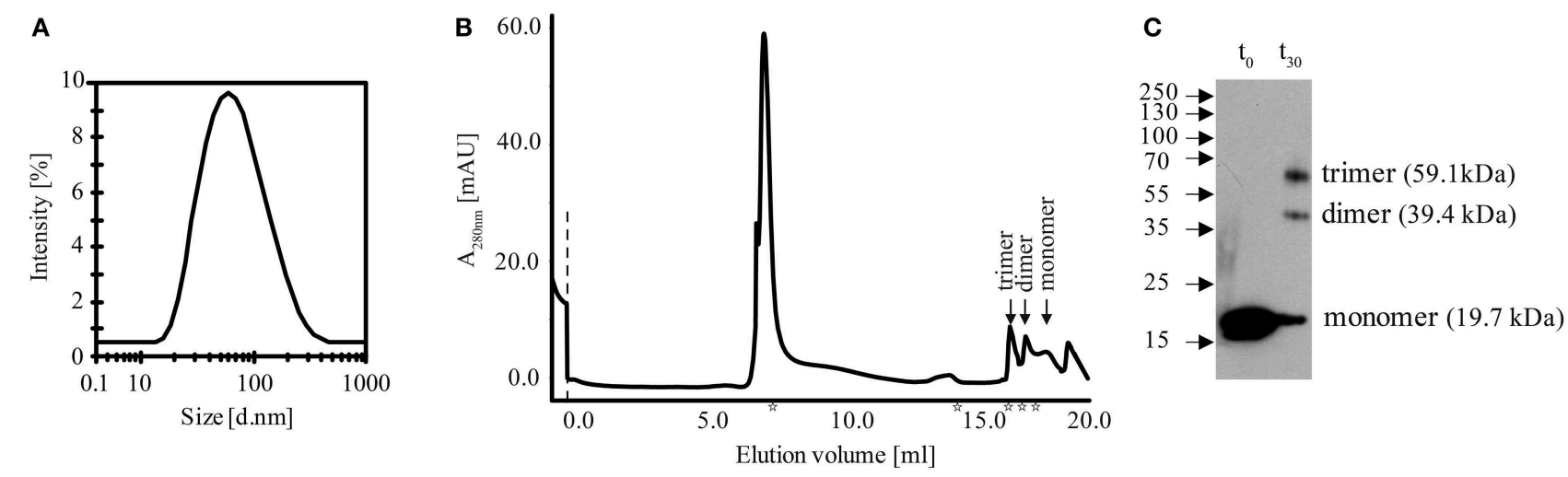

FIGURE 5 | Analysis of the multimeric state of $B$. cereus

C-SpolISA. (A) Dynamic light scattering analysis of C-SpollSA oligomer. Size distribution (by intensity) of $B$. cereus C-SpollSA, at $20^{\circ} \mathrm{C}$, average hydrodynamic radius $=55 \mathrm{~nm}$. (B) Gel filtration of C-SpollSA. The stars indicate the positions at which the following protein standards eluted from the column (left to right): 2000, 450, 66, 45, and $29 \mathrm{kDa}$. (C) Western blot analysis of glutaraldehyde-crosslinked $\mathrm{His}_{6}$-tagged C-SpollSA.

\section{Acknowledgments}

The authors are grateful to Daniela Krajčíková for critical reading of the manuscript, Katarína Muchová for help in designing the pull-down experiments and for critically evaluating the results. The authors would also like to thank Emília Chovancová for technical assistance and Jacob Bauer for helpful comments. This work was supported by Grant 2/0009/13 from the Slovak Academy of Sciences, by a Grant from the Slovak Research and Development Agency under contract APVV-00335-10; APVV14-0181 and by the Research and Development Operational Programme funded by the ERDF (ITMS code: 26240220044).

\section{References}

Adler, E., Barák, I., and Stragier, P. (2001). Bacillus subtilis locus encoding a killer protein and its antidote. J. Bacteriol. 183, 3574-3581. doi: 10.1128/JB.183.12.3574-3581.2001

Ausubel, F. M., Brent, R., Kingston, R. E., Moore, D. D., Seidman, J. G., Smith, J. A., et al. (1987). Protocols in Molecular Biology. New York, NY: Greene Publishing Associates and Wiley-Interscience John Wiley and Sons.

Camacho, A. G., Misselwitz, R., Behlke, J., Ayora, S., Welfle, K., Meinhart, A., et al. (2002). In vitro and in vivo stability of the $2 \zeta 2$ protein complex of the broad host-range Streptococcus pyogenes pSM19035 addiction system. Biol. Chem. 383, 1701-1713. doi: 10.1515/bc.2002.191

Engelberg-Kulka, H., Amitai, S., Kolodkin-Gal, I., and Hazan, R. (2006). Bacterial programmed cell Death and multicellular behavior in bacteria. PLoS Genet. 2:e135. doi: 10.1371/journal.pgen.0020135

Fineran, P. C., Blower, T. R., Foulds, I. J., Humphreys, D. P., Lilley, K. S., and Salmond, G. P. C. (2009). The phage abortive infection system, ToxIN, 
functions as a protein-RNA toxin-antitoxin pair. Proc. Natl. Acad. Sci. U.S.A. 106, 894-899. doi: 10.1073/pnas.0808832106

Florek, P., Levdikov, V. M., Blagova, E., Lebedev, A. A., Škrabana, R., Rešetárová, S., et al. (2011). The structure and interactions of SpoIISA and SpoIISB, a toxin-antitoxin system in Bacillus subtilis. J. Biol. Chem. 286, 6808-6819. doi: 10.1074/jbc.M110.172429

Florek, P., Muchová, K., Pavelč́́ková, P., and Barák, I. (2008). Expression of functional Bacillus SpoIISAB toxin-antitoxin modules in Escherichia coli. FEMS Microbiol. Lett. 278, 177-184. doi: 10.1111/j.1574-6968.2007.00984.x

Gerdes, K., Christensen, S. K., and Løbner-Olesen, A. (2005). Prokaryotic toxin-antitoxin stress response loci. Nat. Rev. Microbiol. 3, 371-382. doi: $10.1038 /$ nrmicro1147

Gerdes, K., Gultyaev, A. P., Franch, T., Pedersen, K., and Mikkelsen, N. D. (1997). Antisense RNA-regulated programmed cell death. Annu. Rev. Genet. 31, 1-31. doi: 10.1146/annurev.genet.31.1.1

Gerdes, K., Rasmussen, P. B., and Molin, S. (1986). Unique type of plasmid maintenance function: postsegregational killing of plasmid-free cells. Proc. Natl. Acad. Sci. U.S.A. 83, 3116-3120. doi: 10.1073/pnas.83.10.3116

Goeders, N., and Van Melderen, L. (2014). Toxin-antitoxin systems as multilevel interaction systems. Toxins (Basel). 6, 304-324. doi: 10.3390/toxins 6010304

Guzman, L. M., Belin, D., Carson, M. J., and Beckwith, J. (1995). Tight regulation, modulation, and high-level expression by vectors containing the arabinose PBAD promoter. J. Bacteriol. 177, 4121-4130.

Hanahan, D. (1983). Studies on transformation of Escherichia coli with plasmids. J. Mol. Biol. 166, 557-580. doi: 10.1016/S0022-2836(83) 80284-8

Hayes, F. (2003). Toxins-antitoxins: plasmid maintenance, programmed cell death, and cell cycle arrest. Science 301, 1496-1499. doi: 10.1126/science.1088157

Hurley, J. M., and Woychik, N. A. (2009). Bacterial toxin HigB associates with ribosomes and mediates translation-dependent mRNA cleavage at A-rich sites. J. Biol. Chem. 284, 18605-18613. doi: 10.1074/jbc.M109.008763

Karimova, G., Pidoux, J., Ullmann, A., and Ladant, D. (1998). A bacterial twohybrid system based on a reconstituted signal transduction pathway. Proc. Natl. Acad. Sci. U.S.A. 95, 5752-5756. doi: 10.1073/pnas.95.10.5752

Laemmli, U. K. (1970). Cleavage of structural proteins during the assembly of the head of bacteriophage T4. Nature 227, 680-685. doi: 10.1038/227680a0

Lehnherr, H., and Yarmolinsky, M. B. (1995). Addiction protein Phd of plasmid prophage $\mathrm{P} 1$ is a substrate of the ClpXP serine protease of Escherichia coli. Proc. Natl. Acad. Sci. U.S.A. 92, 3274-3277. doi: 10.1073/pnas.92.8.3274

Makroczyová, J., Rešetárová, S., Florek, P., and Barák, I. (2014). Topology of the Bacillus subtilis SpoIISA protein and its role in toxin-antitoxin function. FEMS Microbiol. Lett. 358, 180-187. doi: 10.1111/1574-6968.12531

Masuda, H., Tan, Q., Awano, N., Wu, K.-P., and Inouye, M. (2012). YeeU enhances the bundling of cytoskeletal polymers of MreB and FtsZ, antagonizing the CbtA (YeeV) toxicity in Escherichia coli. Mol. Microbiol. 84, 979-989. doi: 10.1111/j.1365-2958.2012.08068.x

Meselson, M., and Yuan, R. (1968). DNA restriction enzyme from E. coli. Nature 217, 1110-1114. doi: 10.1038/2171110a0

Naville, M., Ghuillot-Gaudeffroy, A., Marchais, A., and Gautheret, D. (2011). ARNold: a web tool for the prediction of Rho-independent transcription terminators. RNA Biol. 8, 11-13. doi: 10.4161/rna.8.1.13346

Nicolas, P., Maeder, U., Dervyn, E., Rochat, T., Leduc, A., Pigeonneau, N., et al. (2012). Condition-dependent transcriptome reveals high-level regulatory architecture in Bacillus subtilis. Science 335, 1103-1106. doi: $10.1126 /$ science. 1206848

Nugent, T., and Jones, D. T. (2009). Transmembrane protein topology prediction using support vector machines. BMC Bioinformatics 10:159. doi: 10.1186/14712105-10-159
Pedersen, K., and Gerdes, K. (1999). Multiple hok genes on the chromosome of Escherichia coli. Mol. Microbiol. 32, 1090-1102. doi: 10.1046/j.13652958.1999.01431.x

Rešetárová, S., Florek, P., Muchová, K., Wilkinson, A. J., and Barák, I. (2010). Expression and localization of SpoIISA toxin during the life cycle of Bacillus subtilis. Res. Microbiol. 161, 750-756. doi: 10.1016/j.resmic.2010.09.005

Ruiz-Echevarría, M. J., Giménez-Gallego, G., Sabariegos-Jareño, R., and DíazOrejas, R. (1995). Kid, a small protein of the parD stability system of plasmid $\mathrm{R} 1$, is an inhibitor of DNA replication acting at the initiation of DNA synthesis. J. Mol. Biol. 247, 568-577.

Sambrook, J., Fritsch, E. F., and Maniatis, T. (1989). Molecular Cloning: A Laboratory Manual, 2nd Edn. New York, NY: Cold Spring Harbor Laboratory.

Sayeed, S., Reaves, L., Radnedge, L., and Austin, S. (2000). The stability region of the large virulence plasmid of Shigella flexneri encodes an efficient postsegregational killing system. J. Bacteriol. 182, 2416-2421. doi: 10.1128/JB.182.9.2416-2421.2000

Schägger, H., and von Jagow, G. (1987). Tricine-sodium dodecyl sulfatepolyacrylamide gel electrophoresis for the separation of proteins in the range from 1 to $100 \mathrm{kDa}$. Anal. Biochem. 166, 368-379. doi: 10.1016/00032697(87)90587-2

Schuster, C. F., and Bertram, R. (2013). Toxin-antitoxin systems are ubiquitous and versatile modulators of prokaryotic cell fate. FEMS Microbiol. Lett. 340, 73-85. doi: 10.1111/1574-6968.12074

Solovyev, V., and Salamov, A. (2011). "Automatic annotation of microbial genomes and metagenomic sequences," in Metagenomics and its Applications in Agriculture, Biomedicine and Environmental Studies, ed R. W. Li (New York, NY: Nova Science Publishers), 61-78.

Tsilibaris, V., Maenhaut-Michel, G., Mine, N., and Van Melderen, L. (2007). What is the benefit to Escherichia coli of having multiple toxin-antitoxin systems in its genome? J. Bacteriol. 189, 6101-6108. doi: 10.1128/JB.00527-07

Unterholzner, S. J., Poppenberger, B., and Rozhon, W. (2013). Toxin-antitoxin systems. Mob. Genet. Elements 3:e26219. doi: 10.4161/mge.26219

Van Melderen, L. (2001). Molecular interactions of the CcdB poison with its bacterial target, the DNA gyrase. Int. J. Med. Microbiol. 291, 537-544. doi: 10.1078/1438-4221-00164

Van Melderen, L., and Saavedra De Bast, M. (2009). Bacterial toxinantitoxin systems: more than selfish entities? PLoS Genet. 5:e1000437. doi: 10.1371/journal.pgen.1000437

Wang, X., Lord, D. M., Cheng, H.-Y., Osbourne, D. O., Hong, S. H., SanchezTorres, V., et al. (2012). A new type V toxin-antitoxin system where mRNA for toxin GhoT is cleaved by antitoxin GhoS. Nat. Chem. Biol. 8, 855-861. doi: 10.1038/nchembio.1062

Youngman, P., Perkins, J. B., and Losick, R. (1984). Construction of a cloning site near one end of Tn917 into which foreign DNA may be inserted without affecting transposition in Bacillus subtilis or expression of the transposon-borne erm gene. Plasmid 12, 1-9. doi: 10.1016/0147-619X(84)90061-1

Conflict of Interest Statement: The authors declare that the research was conducted in the absence of any commercial or financial relationships that could be construed as a potential conflict of interest.

Copyright (C 2015 Melničáková, Bečárová, Makroczyová and Barák. This is an openaccess article distributed under the terms of the Creative Commons Attribution License (CC BY). The use, distribution or reproduction in other forums is permitted, provided the original author(s) or licensor are credited and that the original publication in this journal is cited, in accordance with accepted academic practice. No use, distribution or reproduction is permitted which does not comply with these terms. 